\title{
EDITORIAL
}

\section{Pharmacology and psychiatry}

The last 20 years have witnessed a remarkable collusion between psychiatry and hitherto estranged disciplines, pharmacology for one, to the mutual enrichment of each other. The collusion was initially fortuitous, drugs introduced for other purposes being deployed to psychiatry as a consequence of astute clinical reappraisal. For example, chlorpromazine-used first in a premedication mixture-was later employed in treating paranoid schizophrenia and conditions associated with psychomotor activity. Imipramine, one of a number of phenothiazine analogues selected for clinical trial on account of sedative properties, was observed by Kuhn (1958) to be beneficial in depressed patients. Isoniazid and iproniazid, originally used to treat tuberculosis, were noted to elevate mood and subsequently found efficacious for depressive illness. Zeller and Barsky (1952) discovered iproniazid to be a potent monoamine oxidase inhibitor (MAOI) and postulated that its therapeutic efficacy was related to inhibition of monoamine oxidase (MAO) with subsequent increase of brain amines.

These findings have had wondrous repercussions. Pharmaceutical firms, responsible for siring virtually the entire therapeutic 'psychopharmacopoeia', were quick to devise and apply screening tests to identify new compounds of potential value. Newly synthesized compounds, selection for their action on the central nervous system by some general 'screen' such as the Irwin Profile, can be subsequently categorized with a relatively small number of tests according to whether they have properties akin to the MAOI, tricyclic compounds, phenothiazines, butypherones, etc. These tests are as yet empirical in the sense that the drugs are evaluated against only the remotest equivalents of depression or schizophrenia.

The presence of noradrenaline, dopamine, and 5-hydroxytryptamine $(5-\mathrm{HT})$ in the brain, the fact that these amines are inactivated by MAO, the production of a 'model psychosis' with lysergic acid diethylamide (LSD), a substance which mimics some actions of 5-HT and antagonizes others, led to speculation as to the central nervous role of these amines. 'Catecholamine' and 'indoleamine' hypotheses of affective illness were proposed, generating in turn much fundamental research. It is probably more than coincidence that functions integrated by the hypothalamus-for example, appetite, sexual function and drive, are conspicuously involved in affective illness and that the hypothalamus is the site of greatest concentration of brain noradrenaline and 5-HT.

One interpretation of the 'catecholamine' hypothesis attributes mania to 'excess' noradrenaline at central nervous receptors. It must be remembered that, although the synthesis pathway, tyrosine $\rightarrow$ dihydroxyphenylalanine (DOPA) $\rightarrow$ dopamine $\rightarrow$ noradrenaline, had been proposed by Blaschko in 1939, the first and rate-limiting step, conversion of L-tyrosine to L-dopa was characterized only in 1964 by Nagatsu, Levitt, and Udenfriend. A logical treatment for mania would therefore be to exhibit $\alpha$-methyltyrosine, which inhibits tyrosine hydroxylase and so ultimately reduces 'available' noradrenaline. Contrariwise, should depression be due to 'diminished' noradrenaline at central receptors and the pathway for noradrenaline synthesis be intact, it would be reasonable to administer L-dopa, which, given by mouth, unlike dopamine and noradrenaline, reaches the brain. Since DOPA is avidly decarboxylated by DOPA-decarboxylase, widely distributed in the body, L-dopa should be combined with a decarboxylase inhibitor that does not penetrate the blood-brain barrier. Results with DOPA proved disappointing, psychomotor retardation being improved but without amelioration of depressive mood. Moreover, depressive illnesses developed in subjects with Parkinson's disease to whom DOPA was administered.

In practice, the greatest success in treating depression has been with the tricyclic derivatives and to a lesser extent the monoamine oxidase inhibitors. These facts in no way impair the logic of the above 
formulation, since the tricyclic drugs interfere with re-uptake of noradrenaline into noradrenergic nerves, while the monoamine oxidase inhibitors interfere with its deamination, so increasing the amount of transmitter at receptors. There is recent evidence that monomethylated tricyclic compounds-for example, nortriptyline, and desipramine-affect brain noradrenaline metabolism but not that of 5-HT and dopamine, whereas the dimethylated compounds, amitryptiline and imipramine, affect only 5-HT metabolism. Use of tricyclic drugs has been of great help to pharmacologists in studying the fate of noradrenaline after its release (Iversen, 1971), partly by re-uptake into noradrenergic nerves (uptake mechanism $_{1}$ ) and partly by uptake by non-neuronal tissues (uptake mechanism ${ }_{2}$ ).

The 'indoleamine' hypothesis poses a 'deficiency', in depression, of 5-HT at central nervous receptors. Consequently, it would be logical to prescribe the 5-HT precursor, 5-hydroxytryptophan (5-HTP), or the tricyclic drugs which also reduce re-uptake of 5-HT, after its release, back into 5-hydroxytryptaminergic nerves. However, 5-HTP proved ineffective for depression and while there is debate as to the efficacy of tryptophan (Carroll, 1971), there seems agreement that tryptophan potentiates the anti-depressant actions of an MAOI. The 'indoleamine' hypothesis attributes mania to 'excess' 5-HT at central receptors, indicating treatment either by inhibiting the enzyme converting 5-HTP to 5-HT (thereby also interfering with decarboxylation of DOPA) or with a 5-HT antagonist such as methysergide, for which beneficial claims have been made (Dewhurst, 1968). It should be borne in mind that these 'excesses' and 'deficiencies' of central transmitters are extrapolations based on the concentration of transmitter metabolites in the cerebrospinal fluid-or, considerably less pertinent, in the urine.

Research into schizophrenia, a disorder with auditory rather than visual hallucinations, has been dogged by the appearance of chromatographic 'spots' before the eyes. These phenomena, hopefully claimed as metabolites specific to schizophrenia, have proved non-specific on stricter appraisal. Of interest are reports that methylation, a chemical transformation used by the body and resorted to by chemists and pharmacologists in structure-activity studies, is abnormal in schizophrenics. The idea is supported by findings that exhibition of methyl donors such as betaine or methionine leads to exacerbation of schizophrenic 'nuclear' symptoms. There has been speculation as to the relation between schizophrenia, 'model' psychoses induced by hallucinogens, and the effects of hallucinogens and phenothiazines on the central actions of 5-HT and catecholamines. However, chemical theories to account for schizophrenia, although ingenious, are less substantially based than those for affective illness. Amphetamine addiction can produce a clinical picture simulating paranoid schizophrenia (Connell, 1958). Amphetamine acts by releasing intraneuronal noradrenaline and dopamine, by interfering with their re-uptake into nerves, by inhibiting monoamine oxidase, and by virtue of its tryptamine-like properties. Hallucinations, following amphetamine, have been ascribed to its 'tryptamimetic' properties (Vane, 1960) and, indeed, a number of tryptamines are hallucinogenic.

Paradoxically and happily, one facet of the 'drug revolution' is that patients with psychoses become both accessible to and benefit from social and psychological treatments, an approach that would have been regarded as unwarrantable by most psychiatrists two decades ago. Consequently, adherence to pharmacotherapy should in no way neglect the milieu in which the illness throve.

Though claims for a therapeutic miracle have been made, there are no grounds for complacency. There is a need for improved drugs and it is disappointing that, after the breakthrough in the 1950s, there has been consolidation rather than advance. New drugs have appeared but are variants of already accepted tricyclic substances (including phenothiazines). While tricyclic drugs have proved more satisfactory than the monoamine oxidase inhibitors for treating depression, it would be unwise to ignore recent leads-for example, the discovery that monoamine oxidase consists of a number of isoenzymes. This stimulated search for a clinically effective MAOI which did not inhibit the isoenzyme deaminating tyramine, hence avoiding the 'hypertensive crisis' which developed in some patients on MAOI after ingestion of certain foods.

Although to be deplored, such side-effects should be scrutinized closely, since they provide clues as to the mode of action of a drug and often open new areas or research. The 'hypertensive crisis' after eating cheese (Blackwell, 1963) is one such example, and led to considerably greater understanding of 
the pharmacology of foods containing amines and their precursors. Another example-extrapyramidal disorders associated with reserpine, phenothiazine, or butypherone administration-was found to be associated with blockade of central dopamine receptors and increased dopamine turnover in the nervous system. Apart from providing an animal model with which to study extrapyramidal disorders, these findings, combined with the knowledge that Parkinson's disease, due to a variety of causes, is associated with degeneration of and loss of dopamine from striatal neurones, provided impetus for its treatment with DOPA.

Lest it be thought that progress has been confined to psychoses, one might mention the value of propranolol, an antagonist at $\beta$-adrenoreceptors, in patients with anxiety and autonomic symptoms, and of the benzodiazepines in patients with anxiety accompanied by feelings of tension.

These, then, are some of the consequences of the cross-fertilization of psychiatry with pharmacology. There can be little doubt that the liaison has been fruitful; indeed, there are grounds for suspecting concubinage with other disciplines.

E. MARLEY

\section{REFERENCES}

Blackwell, B. (1963). Hypertensive crisis due to monoamineoxidase inhibitors. Lancet, 2, 849-851.

Blaschko, H. (1939). The specific action of L-dopa decarboxylase. Journal of Physiology, 96, 50-51P.

Carroll, B. J. (1971). Monoamine precursors in the treatment of depression. Clinical Pharmacology, 12, 743-761.

Connell, P. H. (1958). Amphetamine Psychosis. Maudsley Monograph No. 5. Chapman \& Hall: London.

Dewhurst, W. G. (1968). Methysergide in mania. Nature, 219, 506-507.

Iversen, L. L. (1971). Role of transmitter uptake mechanisms in synaptic neurotransmission. British Journal of Pharmacology, 41, 571-591.

Kuhn, R. (1958). The treatment of depressive states with
G 22355 (imipramine hydrochloride). American Journal of Psychiatry, 115, 459-464.

Nagatsu, T., Levitt, M., and Udenfriend, S. (1964). Tyrosine hydroxylase. The initial step in norepinephrine biosynthesis. Journal of Biological Chemistry, 239, 2910-2917.

Vane, J. R. (1960). The actions of sympathomimetic amines on tryptamine receptors. In Adrenergic Mechanisms. Ciba Foundation Symposium. Jointly with Committee for Symposia on Drug Action, pp. 356-372. Edited by J. R. Vane, G. E. W. Wolstenholme, and M. O'Connor. Churchill: London.

Zeller, E. A., and Barsky, J. (1952). In vivo inhibition of liver and brain monoamine oxidase by 1-Isonicotinyl-2isopropyl hydrazine. Proceedings of the Society for Experimental Biology and Medicine, 81, 459-461. 